\title{
The Effect of Constant Magnetic Fields on Programmable Shunts
}

\author{
Hüseyin Kurtuldu ${ }^{*}$ (D), Büşra Özgöde ${ }^{1}$
}

\begin{abstract}
Keywords

Hydrocephalus, Programmable Shunts, Magnetic Effect

${ }^{1}$ Department of Biomedical Engineering Başkent University

*Corresponding Author:hkurtuldu@baskent.edu.tr

Manuscript received date: June 21, 2018

Accept Date: August 13, 2018

Published Date: December, 2018.
\end{abstract}

The valve pressure settings of the programmable shunt valves may be changed undesirably in the high magnetic field environments, and in many cases, these changes are not noticeable. Recent studies have shown that this effect increases in parallel with the increasing diversity of permanent magnet containing devices used in daily life. In this study, the effects of the non-ionizing magnetic field produced by devices such as smartphones and headphones on the programmable cerebral shunts were investigated. One of the programmable valves, frequently preferred in the treatment of hydrocephalus, was used in this study. First, the minimum magnetic flux densities that could affect these valves when exposed to an external magnetic field have been determined. Then, the effect of the magnetic flux densities produced by two different earphones and a mobile phone on this valve was investigated. It has been observed that such external magnetic fields can cause undesirable pressure changes on the valves at close distances, and these results suggest that patients should be aware of this effect.

\section{INTRODUCTION}

Hydrocephalus develops as a result of cerebrospinal fluid (CSF) accumulation in ventricles due to the imbalance between the production and absorption of CSF [1]. Since it is not possible to restore the circulation of CSF in the most of the patients, the transfer of fluid from the brain to another body cavity is provided by the systems called shunts. Various shunt types are used in neurosurgical applications. The most preferred of these are the conventional constant pressure shunts and programmable shunts. The pressure settings of the programmable shunt valves can be performed without any surgical or interventional procedure considering the clinical and radiological findings of the patient. The pressure settings of these valves, which are regulated by the magnetic field principle, may vary undesirably and are not mostly noticeable in the high magnetic field environments [2-6, 6-11].

Many studies in the literature show that unwanted opening pressure changes in the programmable valves occur most frequently during MR imaging [12,13]. However, in parallel with the increase in the diversity of devices used in our daily lives, it has been reported that different devices, besides MR imaging, performed this effect in the studies especially in the recent years.

In this study, the effects of the non-ionizing constant magnetic field, produced by devices such as smartphones and headphones, on the programmable cerebral shunts were investigated. According to the measurements of the magnetic flux density, it is seen that the magnetic fields of the shunt valves of these devices are at the levels that can change the pressure settings. For that reason, patients and their relatives should bear in mind that the devices that produce high magnetic fields on the shunt surface may cause undesirable pressure changes in the shunts. 


\section{MATERIALS \& METHOD}

One of the frequently used programmable valves in neurosurgery was selected for the study. The valve can be adjusted to 8 different performance levels, and it is possible to optically measure the performance properties of the valve opening press without requiring a radiological evaluation by means of the transparent layer on the magnets and revolving structure in the shunt. The valve was adjusted to 8 different performance levels to obtain shunt mechanism images by using the zoom-lens camera system (Figure 1). As delineated in the Figure-1, the angles between the tantalum ball-bearing magnet and the fixed reference point are measured according to 8 different performance levels.
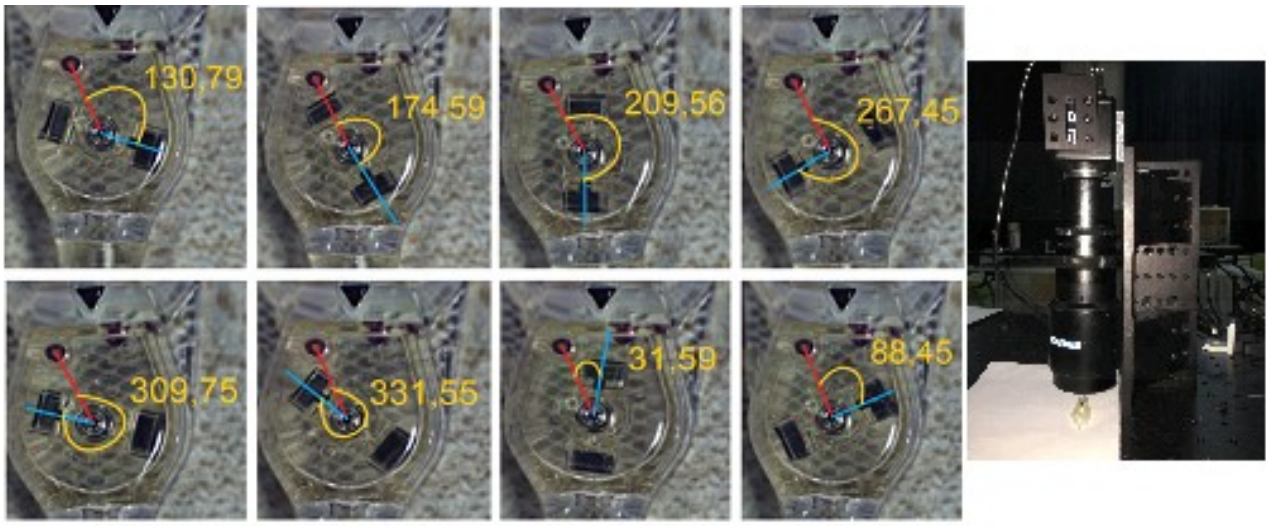

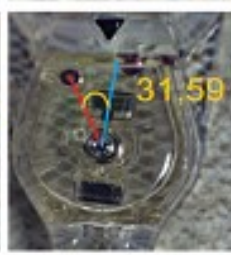

(a) (b)

Figure 1. (a) Images taken using the zoom lens at different performance levels of the valve (b) Zoom-lens camera device

After calculating the angles in the images without the effect of any magnetic field, the valve was exposed to 30 Gauss $(G)$ and $90 \mathrm{G}$ magnetic fields using permanent magnets and the change in the angles at different performance levels were determined. In this study, magnetic field measurements were carried out with a Hall Effect sensor (Honeywell SS49E) to observe the effect of magnetic field created by devices with permanent magnets such as headphones and mobile phones. A scanning system consisting of three-dimensional micro-sensitive translation stages (Psaron HTI) were employed for these measurements. The sensor was mounted on the scanning system and the motion and the sensor are synchronized to measure the magnetic field around the object to be scanned.

The three-dimensional scanning, with the sensor layer mounted on the micro-sensitive stages as indicated in Figure-2, was first performed for the headphone from $1 \mathrm{~mm}$ to $7 \mathrm{~mm}$ from the surface with $1 \mathrm{~mm}$ steps. Similarly, the phone surface is scanned by a movement from $1 \mathrm{~mm}$ to $8 \mathrm{~mm}$ with $0.5 \mathrm{~mm}$ steps. The magnetic flux density measurements were performed by scanning laterally (x-y plane) the selected regions of iPhone 6 Plus, placed under the sensor at different heights (z-axis). A smartphone (iPhone 6 Plus) and 2 different headphones (Apple EarPods and Philips SHE1350) have been tested for this study. 


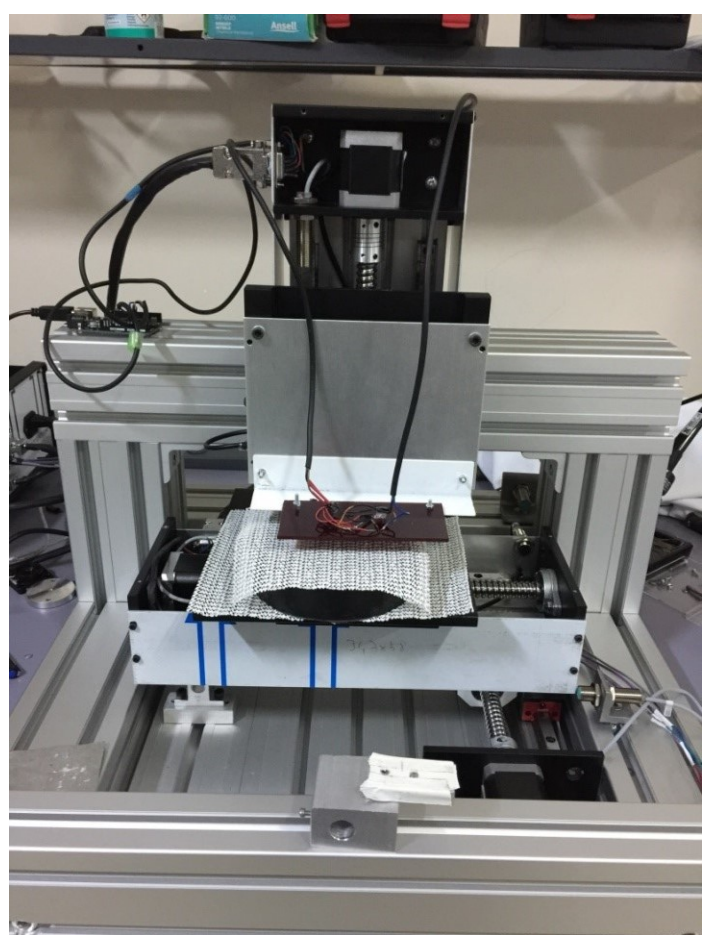

Figure 2. The three-dimensional measurement system with micro-sensitive translational stages

\section{FINDINGS}

Figure-3 shows the angles calculated with respect to the valve reference point at 8 different performance levels.

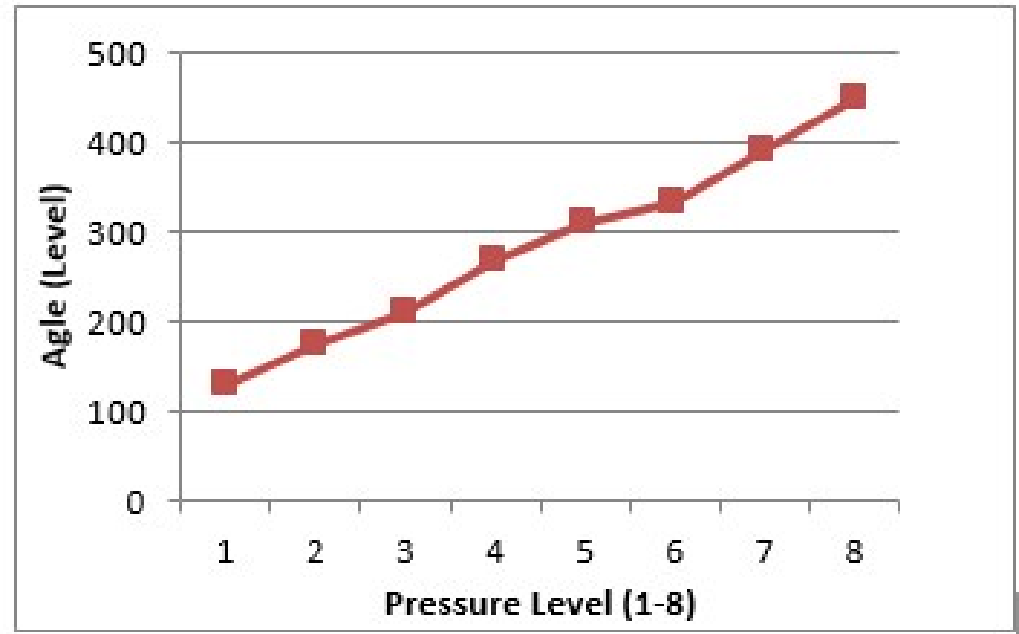

Figure 3. The angles calculated from the camera images according to the valve pressure level

The linear relationship between the angle and the pressure obtained by analyzing the camera images was found as,

$$
\theta=1,3314 P+106,22
$$

, where $\theta$ is the angle between the magnet and the reference point; and $\mathrm{P}$ is the valve opening pressure. Later, the valve was exposed to $30 \mathrm{G}$ and $90 \mathrm{G}$ magnetic fields at the 4th performance level $\left(110 \mathrm{~mm} \mathrm{H}_{2} \mathrm{O}\right)$ and the 5 th performance level (145 $\mathrm{mm} \mathrm{H}_{2} \mathrm{O}$ ) to examine the effect of the permanent magnet on the valve. Immediately after the valve was exposed to the magnetic field, the images were captured by the camera and the change of the magnet angle with respect to the reference point was recorded. The angles measured at the fourth performance level of the valve and at $0 \mathrm{G}, 30 \mathrm{G}$ and $90 \mathrm{G}$ magnetic fields are shown in Figure-4. Figure-5 describes how the angle for the fifth performance level changes over $30 \mathrm{G}$ and $90 \mathrm{G}$ magnetic fields. 

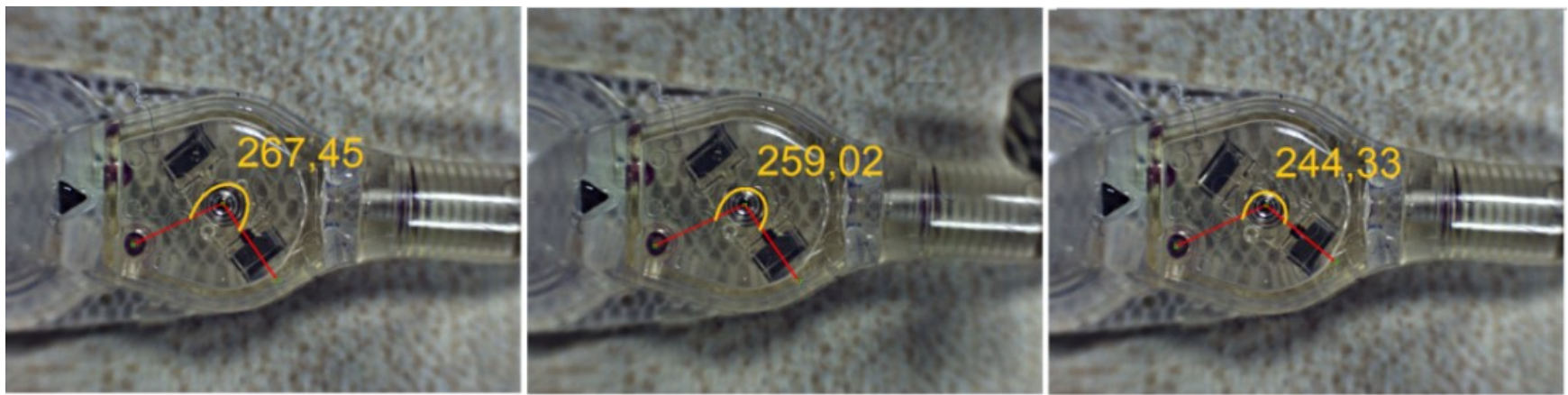

Figure 4. $\left(110 \mathrm{~mm} \mathrm{H}_{2} \mathrm{O}\right)$ a) $0 \mathrm{G} \mathrm{b}$ ) $\left.30 \mathrm{G} \mathrm{c}\right) 90 \mathrm{G}$ the magnetic field effects at the fourth performance level
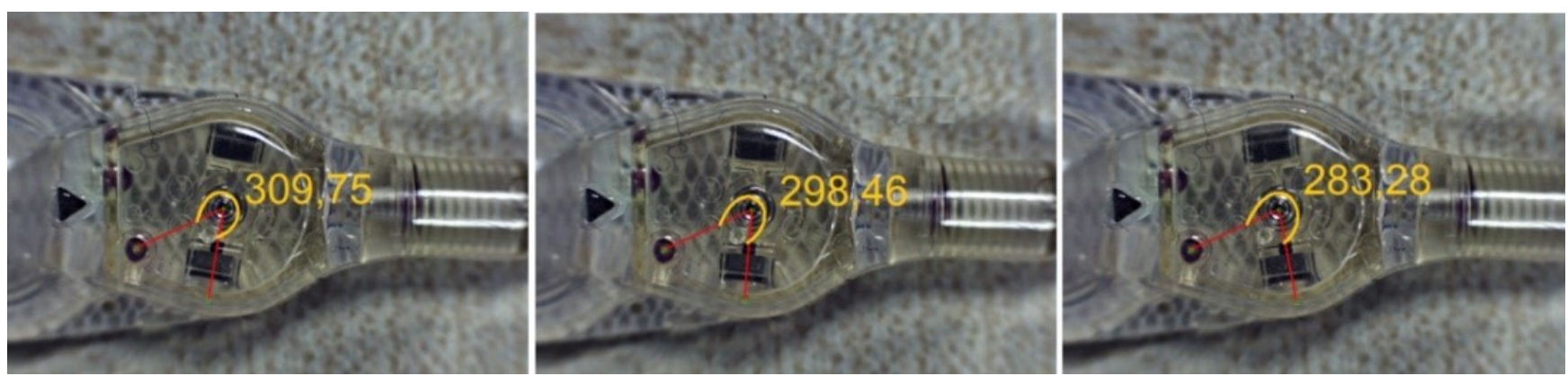

Figure 5. $\left(145 \mathrm{mmH}_{2} \mathrm{O}\right.$ ) a) $\left.\left.0 \mathrm{G} \mathrm{b}\right) 30 \mathrm{G} \mathrm{c}\right) 90 \mathrm{G}$ the magnetic field effects at the fifth performance level

Comparisons were made with a low level of the tested performance level to determine whether the angle changes would change the valve performance level. '3rd Level Opening Pressure' and '4th Level Opening Pressure' are shown together in Figure-6. The graph contains $\mp$ tolerances according to the tolerance percentages obtained from the valve datasheet. The angle alterations that occurred when the valve exposed to the $30 \mathrm{G}$ and $90 \mathrm{G}$ magnetic field are converted to pressure information according to the linear curve function (equation (1)) and added on the same graph. Although the opening pressure value has decreased, it has not reached the performance level of the previous level as it is seen in Figure-6.

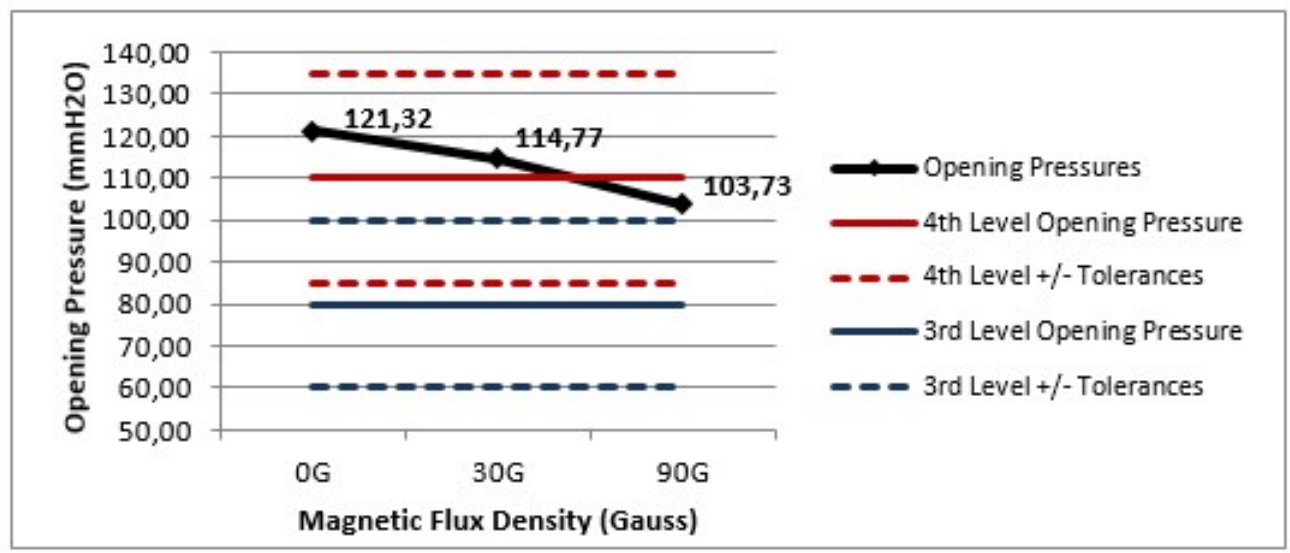

Figure 6. Comparison of the opening pressures of the third and fourth performance levels

'4th Level Opening Pressure' and '5th Level Opening Pressure' are shown together in Figure-7. The graph contains ₹ tolerances according to the tolerance percentages obtained from the valve datasheet. The angle variations that occurred when the valve exposed to the $30 \mathrm{G}$ and $90 \mathrm{G}$ magnetic field are converted to pressure information according to the linear curve function (equation (1)) and added on the same graph. Although the angle level has decreased, it has not reached the distance of 4th performance level when the $30 \mathrm{G}$ magnetic field is applied at the 5th performance level as it is seen in Figure-7. However, when the $90 \mathrm{G}$ magnetic field is applied, the 4th performance level appears within the $\mp$ tolerance distance. 


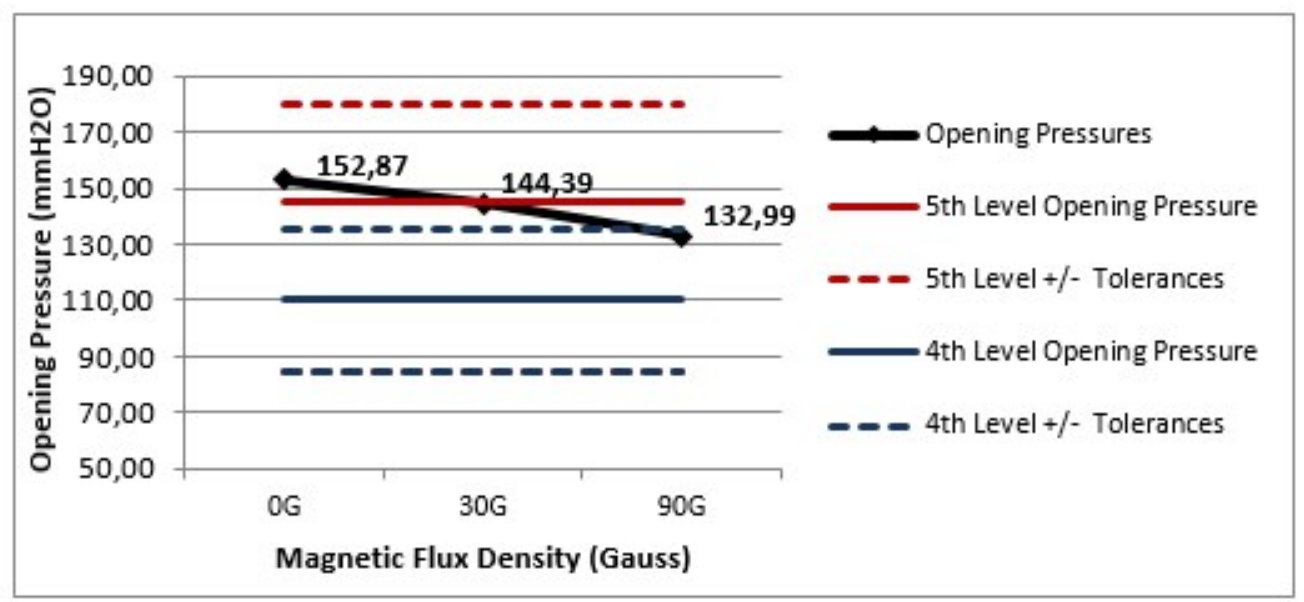

Figure 7. Comparison of the opening pressures of the fourth and fifth performance levels

*The 5th level -deviation is not seen because of the -deviation of the 5th level and 4th level pressure overlap at the 110 $\mathrm{mm} \mathrm{H}_{2} \mathrm{O}$ level

\subsection{Magnetic flux density measurement of the headphones}

The magnetic flux densities on the surface of the Apple EarPods headphone are depicted in Figure-8. As represented, the magnetic flux density reaches to a maximum of $169 \mathrm{G}$ in the center of the headphone.

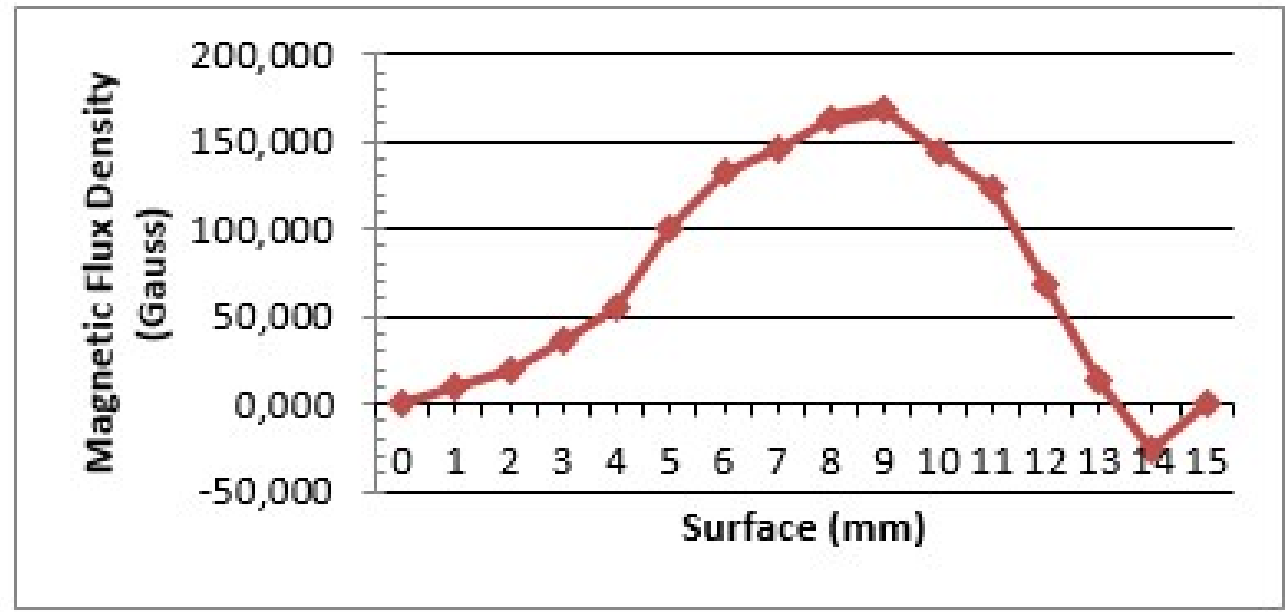

Figure 8. The magnetic flux density on the inner surface of the Apple headphone

The magnetic flux densities on the Philips SHE1350 headphone surface are delineated in Figure-9. As shown, the magnetic flux density reaches up to $231,4 \mathrm{G}$ in the center of the headphone. 
Table 1. Maximum magnetic flux densities (M.F.D.) as a function of distance from the headphone surfaces

\begin{tabular}{|l|l|l|l|l|l|l|l|l|}
\cline { 2 - 9 } \multicolumn{1}{c|}{} & \multicolumn{7}{c|}{$\begin{array}{c}\text { Max } \\
\text { M.F.D. } \\
(\mathrm{G})\end{array}$} \\
\cline { 2 - 9 } & $(0 \mathrm{~mm})$ & $(1 \mathrm{~mm})$ & $(2 \mathrm{~mm})$ & $(3 \mathrm{~mm})$ & $(4 \mathrm{~mm})$ & $(5 \mathrm{~mm})$ & $(6 \mathrm{~mm})$ & $(7 \mathrm{~mm})$ \\
\hline Apple Headphone & 169,30 & 120,33 & 68,06 & 45,20 & 35,40 & 28,80 & 19,06 & 15,80 \\
\hline Philips Headphone & 231,40 & 166,06 & 94,20 & 58,26 & 45,20 & 35,40 & 22,30 & 19,66 \\
\hline
\end{tabular}

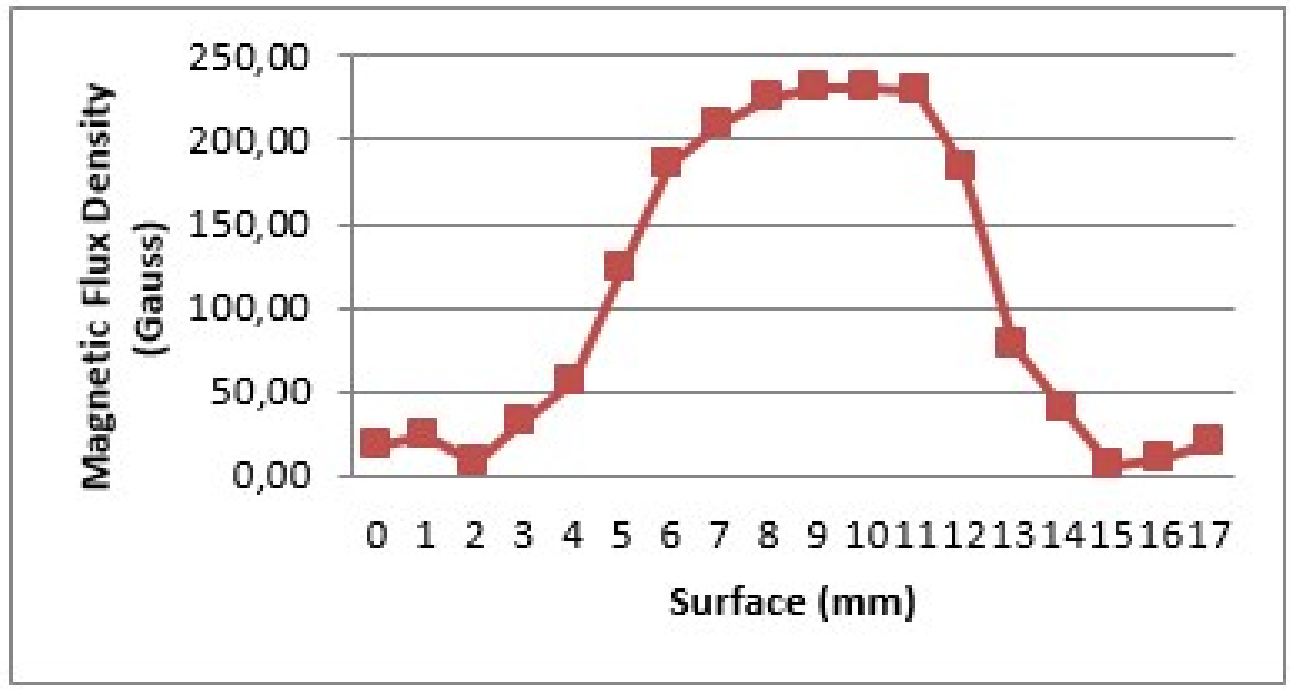

Figure 9. Magnetic flux density on the inner surface of the Philips headphone

In order to observe how the magnetic flux density changes when moving away from the headphones, the measurements were carried out by moving the sensor from the surface with $1 \mathrm{~mm}$ steps. The maximum flux densities measured for the two headphones as a function of the distance from the surface are listed in Table-1.

\section{2 iPhone 6 Plus smartphone magnetic flux density measurement}

The colormaps generated by magnetic flux densities measured laterally in increments of $0.5 \mathrm{~mm}$ from the surface on the reverse side of the loudspeaker part of the mobile phone are shown in Figure-10. The maximum flux density reaches up to $140 \mathrm{G}$ at the nearest distance $(1 \mathrm{~mm})$ to the phone surface. Measured at $8 \mathrm{~mm}$ distance, this value decreases to $17 \mathrm{G}$. The maximum flux density is due to the phone's built-in speaker with a permanent magnet. In addition, a zoom lens containing another permanent magnet on the camera side of the mobile phone also causes high magnetic flux density. 
Table 2. Maximum magnetic flux densities as a function of distance away from four different regions of the mobile phone

\begin{tabular}{l|c|c|c|c|c|c|c|c|c|}
\cline { 2 - 9 } & \multicolumn{7}{c}{ Distance } \\
& 1 & 2 & 3 & 4 & 5 & 6 & 7 & 8 \\
\hline & 218,33 & 162,80 & 126,86 & 90,93 & 68,06 & 55,00 & 45,20 & 41,93 \\
\hline $\begin{array}{l}\text { Loudspeaker } \\
\text { Part } \\
\begin{array}{l}\text { Front } \\
\text { Side }\end{array}\end{array}$ & 141,00 & 108,33 & 78,93 & 59,33 & 49,53 & 39,73 & 36,46 & 33,20 \\
\hline $\begin{array}{l}\text { Loudspeaker } \\
\text { Part Reverse Side }\end{array}$ & 114,86 & 82,20 & 59,33 & 65,86 & 39,73 & 39,73 & 39,73 & 36,46 \\
\hline $\begin{array}{l}\text { Camera Part } \\
\text { Reverse } \\
\text { Side }\end{array}$ & 58,26 & 41,93 & 35,40 & 25,60 & 22,33 & 15,80 & 15,80 & 12,53 \\
\hline $\begin{array}{l}\text { Camera } \\
\text { Part Front Side }\end{array}$ & & & & & & & & \\
\hline
\end{tabular}

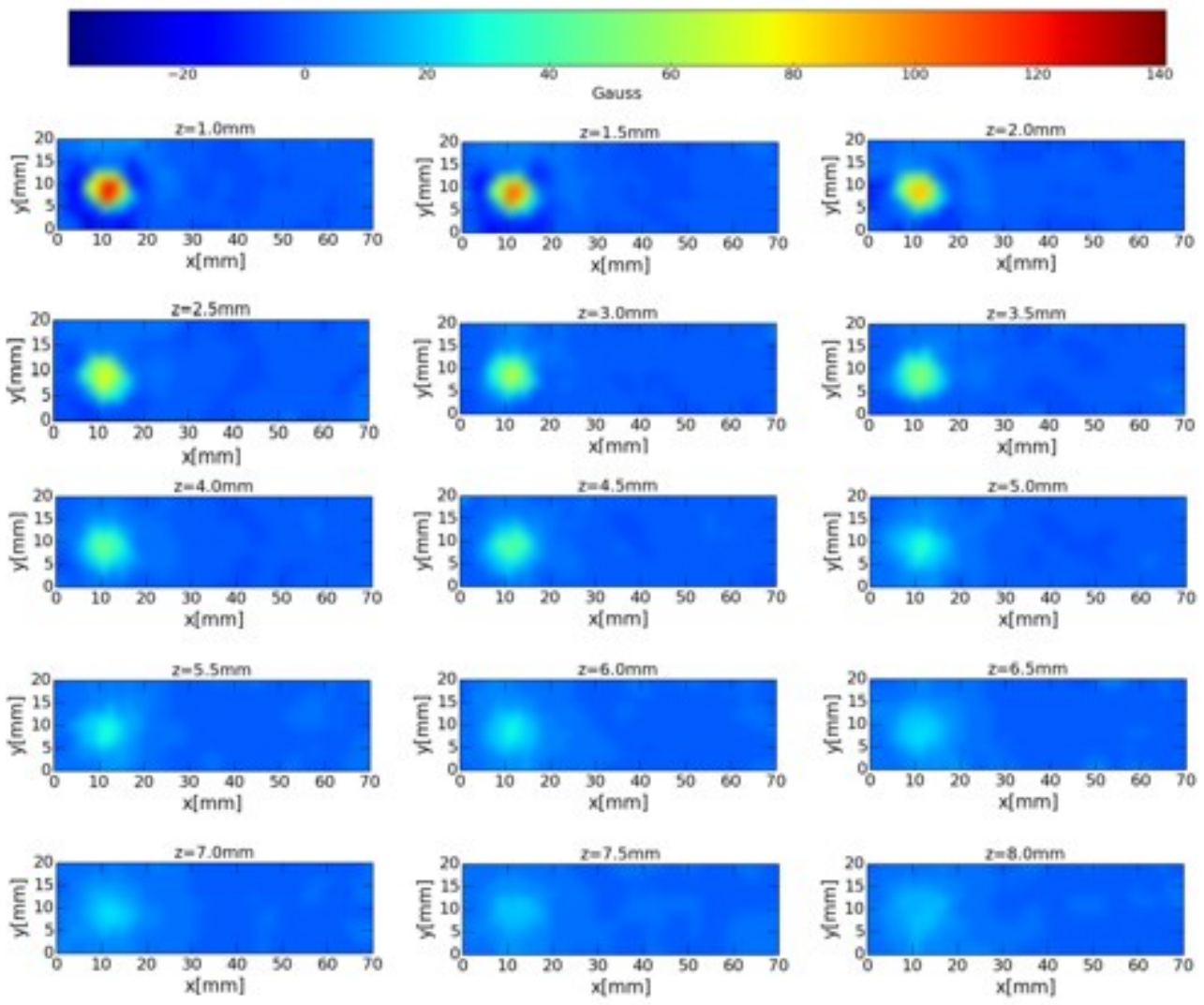

Figure 10. The magnetic maps of iPhone 6 Plus loudspeaker part at different distances on the reverse side

Scanning measurements have also been performed at the front side of the loudspeaker part, the reverse side of the camera part and the front side of the camera part. After generating the magnetic colormaps for all regions, it has also been investigated how the maximum magnetic flux density changes as a function of the distance from the surfaces of the regions. Table-2 shows the maximum magnetic flux densities obtained at different distances (from $1 \mathrm{~mm}$ to $8 \mathrm{~mm}$ ) for four regions of the phone. The magnetic field is more than $90 \mathrm{G}$ at $4 \mathrm{~mm}$ from the loudspeaker at the front of the mobile phone and over $40 \mathrm{G}$ at $8 \mathrm{~mm}$ distance.

\section{DISCUSSION}


Table 3. Reported magnetic flux densities affecting different types of shunt valves [14]

\begin{tabular}{|l|c|}
\hline Valve & Magnetic Flux Density $(\mathrm{G})$ \\
\hline Hakim & 175 \\
\hline Sophy & 400 \\
\hline Strata & 60 \\
\hline
\end{tabular}

\subsection{The effect of magnetic field on the programmable shunt valves}

The positional changes to the performance level of the magnets in the shunt valves were investigated in order to examine the effects of environmental magnetic fields on the programmable shunt valves. The angles corresponding to the performance levels were determined by calculating the angles of the magnet positions with respect to a reference point, and a linear relationship between the angle and the valve opening pressure was obtained. Thus, the position change caused by the effect of the magnetic flux density was expressed in terms of opening pressure change. The valve was exposed to $30 \mathrm{G}$ and $90 \mathrm{G}$ magnetic field at the 4th and 5th performance levels for the determination of these pressure changes. It was observed that as the magnetic field increases at the fourth performance level, the position of the valve magnet approaches to the third performance level. For this reason, the changes in the valve opening pressure were shown on a graph with the effect of the magnetic field applied to the 4th performance levels. As delineated in Figure-5, the opening pressures on the influence of the magnetic fields applied at the 4th performance level did not exceed the upper and lower limits of the 4th performance level and approached to the upper limit of the 3rd performance level but did not reach the tolerance range of this level. As the magnetic field was increased at the 5th performance level, it appeared that the valve magnet position approaches to the 4th performance level. For this reason, the valve opening pressure changes with the magnetic field effect applied at the 5th performance level were shown in Figure-6. Although the angle of the magnet under the $90 \mathrm{G}$ magnetic field at 5th performance level did not exceed the lower limit of 5th performance level, it exceeded the upper limit of 4th performance level. In this case, it may be considered that the field of magnitude $90 \mathrm{G}$ and above can lower the valve setting at the 5th performance level into the 4th performance level. This may cause that the valve set its opening pressure is set at $110 \mathrm{~mm} \mathrm{H}_{2} \mathrm{O}$ instead of $145 \mathrm{mmH}_{2} \mathrm{O}$, resulting in less BOS drainage. However, it is not possible to say that the performance level setting of this valve has been completely modified since the 5th performance level did not exceed the tolerance range.

\subsection{The effect of headphones on the shunt valves}

The headphones are closer to the shunt valve than the mobile phone because they are inserted into the inner ear. The maximum magnetic flux density measured for Apple and Philips headphones were $169.3 \mathrm{G}$ and $231.4 \mathrm{G}$, respectively. Such magnetic flux densities produced by headphones are too high, and cannot be underestimated. Considering the effect of the relatively low magnetic field $(90 \mathrm{G})$ on the opening pressure, the magnetic field, which is almost twice the magnitude, may cause serious changes in the opening pressure levels of the valves. For this reason, programmable shunt patients should be aware of the use of high-field-producing headphones.

\subsection{The effect of iPhone smartphone on the shunt valves}

Smartphones have four specific regions that create the high magnetic flux density: the camera, headphone, microphone, and the powerful speaker of the phone. The maximum magnetic flux density of $218 \mathrm{G}$ was observed in the speaker region. The magnetic field generated by the mobile phone does not make a difference in the pressure setting as long as the phone is not in the close proximity of the shunt valve. However, the magnetic flux density at very close distances ( $2 \mathrm{~mm}$ and less) from the phone surface may still be above $90 \mathrm{G}$ and may change the pressure setting.

Nomura et al. [14] analyzed the effect of mobile phones on 3 different valves which are not protected by $3 \mathrm{~T}$ magnetic field. They have reported that the density at which the Strata valve was affected was 60 G, the Hakim valve was 175 G, and the Sophy valve was $400 \mathrm{G}$ (Table-3). Strata is the most preferred valve brand in the world by physicians. The distance between the valve and the mobile phones should be particularly important for these valves, since the magnetic flux density of $60 \mathrm{G}$ can be observed at a distance of 4-5 $\mathrm{mm}$ from the iPhone surface. In addition, as technology improves, parts such as zoom lenses and stronger permanent magnet speakers are used to get better sound and better quality images on smartphones. For this reason, it is expected that future devices will produce higher magnetic flux densities.

\section{CONCLUSION}

In this study, the effects of magnetic fields generated by devices such as headphones and mobile phones, which became part of everyday life, on programmable shunt valves were investigated. The experiments were performed on transparent shunts which allow optical inspection of valve settings in visible spectrum. However, comparable effects are also expected in opaque shunts as the pressure adjustment mechanism of almost all shunt types on the market is similar. As the results suggested, magnetic 
fields produced by mobile phones and headphones may cause changes in pressure levels although some valve manufacturers claim that the valve opening pressure will not change even at 3 Tesla MR imaging.

Due to the cost of a new generation of 3T MR compatible shunt valves, earlier types of shunt valves (more susceptible to magnetic fields) are used in many countries, including Turkey. Therefore, it should be kept in mind that some devices have reversible effects without creating any radiological evidence on the programmable shunts, and any surgeon implanting these valves and any patient carrying them should be aware of the possible effects. Patients with severe hydrocephalus can replace devices that produce critical magnetic fields with devices that emit fewer magnetic fields to reduce such potential hazards. Also, if patients use non-implanted side of their heads to respond to phone calls, the magnetic field in the shunt valve will fall to an ineffective level as the distance between the shunt valve and the phone increases.

\section{References}

[1] A. Aschoff, P. Kremer, B. Hashemi, and S. Kunze, "The scientific history of hydrocephalus and its treatment," Neurosurgical review, vol. 22, no. 2-3, pp. 67-93, 1999.

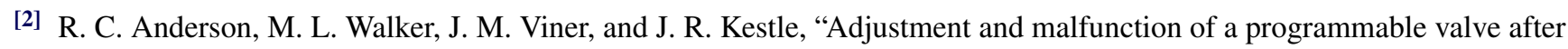
exposure to toy magnets: case report," Journal of Neurosurgery: Pediatrics, vol. 101, no. 2, pp. 222-225, 2004.

[3] S. Utsuki, S. Shimizu, H. Oka, S. Suzuki, and K. Fujii, "Alteration of the pressure setting of a codman-hakim programmable valve by a television," Neurologia medico-chirurgica, vol. 46, no. 8, pp. 405-407, 2006.

[4] M. R. Guilfoyle, H. Fernandes, and S. Price, "In vivo alteration of strata valve setting by vagus nerve stimulator-activating magnet," British journal of neurosurgery, vol. 21, no. 1, pp. 41-42, 2007.

[5] S. Ozturk, H. Cakin, H. Kurtuldu, O. Kocak, F. S. Erol, and M. Kaplan, "Smartphones and programmable shunts: Are these indispensable phones safe and smart?" World neurosurgery, vol. 102, pp. 518-525, 2017.

[6] A. Lavinio, S. Harding, F. Van Der Boogaard, M. Czosnyka, P. Smielewski, H. K. Richards, J. D. Pickard, and Z. H. Czosnyka, "Magnetic field interactions in adjustable hydrocephalus shunts," 2008.

[7] H. S. Spader, L. Ratanaprasatporn, J. F. Morrison, J. A. Grossberg, and G. R. Cosgrove, "Programmable shunts and headphones: Are they safe together?" Journal of Neurosurgery: Pediatrics, vol. 16, no. 4, pp. 402-405, 2015.

${ }^{[8]}$ I. AlTuN, K. Z. YUKSEL, and T. Mert, "Impact of magnetic field on pressures of programmable cerebrospinal fluid shunts: An experimental study," Turkish neurosurgery, vol. 27, no. 6, 2016.

[9] M. Lefranc, J. Y. L. Ko, J. Peltier, A. Fichten, C. Desenclos, J.-M. Macron, P. Toussaint, D. Le Gars, and M. Petitjean, "Effect of transcranial magnetic stimulation on four types of pressure-programmable valves," Acta neurochirurgica, vol. 152, no. 4, pp. 689-697, 2010.

[10] Y. He, R. K. Murphy, J. L. Roland, and D. D. Limbrick, "Interactions between programmable shunt valves and the ipad 3 with smart cover," Child's Nervous System, vol. 29, no. 4, pp. 531-533, 2013.

[11] T. J. Zuzak, B. Balmer, D. Schmidig, E. Boltshauser, and M. A. Grotzer, "Magnetic toys: forbidden for pediatric patients with certain programmable shunt valves?" Child's Nervous System, vol. 25, no. 2, p. 161, 2009.

${ }^{[12]}$ F. G. Shellock, S. F. Wilson, and C. P. Mauge, "Magnetically programmable shunt valve: Mri at 3-tesla," Magnetic resonance imaging, vol. 25, no. 7, pp. 1116-1121, 2007.

[13] T. Inoue, Y. Kuzu, K. Ogasawara, and A. Ogawa, "Effect of 3-tesla magnetic resonance imaging on various pressure programmable shunt valves," Journal of Neurosurgery: Pediatrics, vol. 103, no. 2, pp. 163-165, 2005.

[14] S. Nomura, H. Fujisawa, and M. Suzuki, "Effect of cell phone magnetic fields on adjustable cerebrospinal fluid shunt valves," Surgical neurology, vol. 63, no. 5, pp. 467-468, 2005. 